04,09

\title{
Экспериментальное исследование и ab initio расчет свойств Sc-, In-допированных титанатов висмута со структурой типа пирохлора
}

\author{
(C) А.Г. Краснов ${ }^{1}$, И.Р. Шеин ${ }^{2}$, И.В. Пийр ${ }^{1}$ \\ ${ }^{1}$ Институт химии КомиНЦ УрО РАН, \\ Сыктывкар, Россия \\ ${ }^{2}$ Институт химии твердого тела УрО РАН, \\ Екатеринбург, Россия \\ E-mail: alexey-krasnov@rambler.ru
}

(Поступила в Редакцию 24 августа 2016 г.)

\begin{abstract}
С использованием $a b$ initio расчетов получены данные о структурных, электронных, оптических свойствах титаната висмута со структурой типа пирохлора и соединений с замещением атомами скандия, индия позиций висмута, титана. Результаты теоретических расчетов согласуются с экспериментально полученными структурными и оптическими характеристиками синтезированных допированных соединений. Показано, что замещение атомов висмута в структуре пирохлора атомами Sc или In энергетически выгодно. На основании данных оптической спектроскопии образцов определены величины энергии, соответствующие прямому и непрямому переходам в Sc-, In-допированных титанатах висмута, которые находятся в согласии с теоретически рассчитанными значениями.
\end{abstract}

Работа выполнена при финансовой поддержке РФФИ (проекты № 15-03-09173 А, 16-33-00153 мол_а).

Исследования выполнены с использованием оборудования Центра коллективного пользования „Химия“ Института химии Коми НЦ УрО РАН.

DOI: 10.21883/FTT.2017.03.44158.256

\section{1. Введение}

Соединения на основе титаната висмута со структурой типа пирохлора перспективны как материалы с высокой диэлектрической проницаемостью, малым током утечки [1-4] и смешанные электронно-ионные проводники [5,6]. Кроме того, $\mathrm{Bi}_{2} \mathrm{Ti}_{2} \mathrm{O}_{7}$ получаемый в наноразмерном состоянии, обладает фотокаталитической активностью в видимой области спектра [7-10].

Кристаллическая структура пирохлора $A_{2} B_{2} \mathrm{O}_{6} \mathrm{O}^{\prime}$ $\left(\mathrm{O}\right.$ и $\mathrm{O}^{\prime}$ - неэквивалентные атомы кислорода) описывается пространственной группой $F d \overline{3} m$ (№ 227) и может быть представлена как взаимопроникновение двух подрешеток: $\mathrm{BO}_{6}$-октаэдров, соединенных вершинами, и $A_{2} \mathrm{O}^{\prime}[11]$. В настоящей работе для проведения $a b$ initio расчетов использовалась $A$-центрированная конфигурация: катион $A(\mathrm{Bi})$ помещался в позицию $16 c(0 ; 0 ; 0)$, катион $B(\mathrm{Ti})$ - в позицию $16 d(0.5 ; 0.5 ; 0.5)$, анион кислорода $\mathrm{O}$ - в позицию $48 f(0.125 ; 0.125 ; x)$, анион кислорода $\mathrm{O}^{\prime}$ — в позицию $8 a(0.125 ; 0125 ; 0.125)$ [12].

Ионный радиус висмута (1.17 $\AA$ [13]) является одним из самых больших среди радиусов $A$-катионов в семействе соединений со структурой типа пирохлора. Это приводит к тому, что $\mathrm{Bi}_{2} \mathrm{Ti}_{2} \mathrm{O}_{7}$ не попадает в „поле стабильности пирохлоров“ - область соотношений радиусов $A$ - и $B$-катионов, соответствующую образованию устойчивой кристаллической структуры пирохлора [11]. Кроме того, ранее было показано [14-16], что Вi $6 s$-неподеленная электронная пара создает пространственные затрудения для расположения ионов, что может приводить к смещениям атомов висмута из высокосимметричных позиций в позиции $96 h, 96 g$ или $192 i$ и вследствие этого к смещениям атомов $\mathrm{O}^{\prime}$. Такие особенности кристаллического строения являются причинами термической нестабильности стехиометрической фазы $\mathrm{Bi}_{2} \mathrm{~T}_{2} \mathrm{O}_{7}$, что ограничивает возможность его получения в виде плотной керамики с помощью твердофазной реакции и дальнейшее практическое использование $[1,17]$.

Стабильность кристаллической структуры пирохлора титаната висмута может быть достигнута путем замещения части катионов висмута катионами других элементов с меньшим ионным радиусом. Так, с использованием реакции твердофазного синтеза были получены термостабильные титанаты висмута со структурой пирохлора, допированные атомами $\mathrm{Cr}, \mathrm{Mn}, \mathrm{Fe}, \mathrm{Cu}, \mathrm{Zn}, \mathrm{Y}$ и $\mathrm{Nd}$, и изучены их электрофизические свойства [5,6,18-25].

Ранее с помощью $a b$ initio расчетов были получены данные об электронной структуре и оптических свойствах стехиометрического $\mathrm{Bi}_{2} \mathrm{Ti}_{2} \mathrm{O}_{7}[1,16,26-32]$. Авторы упомянутых работ показали, что это соединение относится к диэлектрикам с прямым электронным переходом и величиной запрещенной щели 2.6-2.85eV [29,32]. Позже было выявлено, что при замещении части атомов Ti 3d-элементами ( $\mathrm{Fe}, \mathrm{Ni}, \mathrm{Cr}, \mathrm{Mn}$ и V) происходит формирование новых энергетических состояний внутри запрещенной щели и длинноволновый сдвиг в спектрах поглощения (по сравнению со стехиометрическим $\mathrm{Bi}_{2} \mathrm{Ti}_{2} \mathrm{O}_{7}$ ) [29]. В работе [33] авторы сообщают, что замещение атомов $\mathrm{Bi}$ атомами $\mathrm{Cr}, \mathrm{Mn}$ и $\mathrm{Fe}$ в пирохлоре 
$\mathrm{Bi}_{2} \mathrm{Ti}_{2} \mathrm{O}_{7}$ энергетически более предпочтительно, чем замещение этими атомами позиций Ті. В результате допирования происходит уменьшение величины запрещенной щели в среднем на $0.3 \mathrm{eV}$.

Ранее нами были изучены области изоморфного замещения в Sc-, In-допированных титанатах висмута co структурой типа пирохлора $\mathrm{Bi}_{2-y} \mathrm{M}_{x} \mathrm{Ti}_{2} \mathrm{O}_{7-1.5 y+1.5 x}$ $(M=\mathrm{Sc}, \quad \mathrm{In} ; \quad y=0.4$ при $x=0.2-0.6 ; y=0.6$ при $x=0.4-0.6)$ и показано распределение допанта по катионным позициям [34,35].

В настоящей работе представлены результаты изучения оптических свойств синтезированных титанатов висмута со структурой типа пирохлора, допированных скандием, индием (25 at.\%), которые сопоставляются с данными $a b$ initio квантово-химических расчетов структурных, электронных и оптических свойств стехиометрического пирохлора $\mathrm{Bi}_{2} \mathrm{Ti}_{2} \mathrm{O}_{7}$ и допированного в позиции висмута (титана) $\mathrm{Bi}_{1.5} M_{0.5} \mathrm{Ti}_{2} \mathrm{O}_{7}, \mathrm{Bi}_{2} \mathrm{Ti}_{1.5} M_{0.5} \mathrm{O}_{7}$ $(M=\mathrm{Sc}, \mathrm{In})$.

\section{2. Эксперимент}

Синтез Sc-, In-содержащих титанатов висмута co структурой типа пирохлора $\mathrm{Bi}_{1.5} M_{0.5} \mathrm{Ti}_{2} \mathrm{O}_{7}$, $\mathrm{Bi}_{2} \mathrm{Ti}_{1.5} M_{0.5} \mathrm{O}_{7}(M=\mathrm{Sc}, \mathrm{In})$ был выполнен твердофазным способом [34,35]. Методом рентгенофазового анализа исследовался фазовый состав образцов с помощью дифрактометра Shimadzu XRD-6000 (CuK $K_{\alpha}$-излучение, $\lambda=1.54056 \AA)$. Для целей структурного анализа выполнена съемка дифрактограмм в угловом интервале от 10 до $100^{\circ}$ с шагом $0.05^{\circ}$ и временем экспозиции $2 \mathrm{~s}$. Элементный микроанализ проведен методом энергодисперсионной спектроскопии (EDS) на сканирующем электронном микроскопе VEGA-3M с использованием приставки $X$-act (Oxford Instruments). Полнопрофильный анализ рентгенограмм осуществлен с помощью пакета программ FullProf [36]. Значения пикнометрической плотности определены согласно ГОСТ 2211-65 на воздухе [37]. Дифференциальная сканирующая калориметрия (ДСК) выполнена на приборе Netzsch STA 409 PC/PG в области температур $25-1300^{\circ} \mathrm{C}$ со скоростью нагрева $5^{\circ} \mathrm{C} / \mathrm{min}$ в воздушной атмосфере. Спектры поглощения записаны с помощью спектрофотометра Perkin Elmer Lambda 1050 в диапазоне 190-1100 nm с шагом $5 \mathrm{~nm} \mathrm{c}$ использованием интегрирующей сферы $150 \mathrm{~mm}$ InGaAs.

\section{3. Модели и метод расчета}

Расчеты проведены в рамках теории DFT (density functional theory) методом проекционных присоединенных волн PAW (projector augmented wave) по программе Vienna $A b$ initio Simulation Package (VASP) [38,39] с обобщенной градиентной аппроксимацией GGA (generalized gradient approximation) обменно-корреляционного функционала в форме РВE [40].

В качестве начальных использовались данные для стехиометрического $\mathrm{Bi}_{2} \mathrm{Ti}_{2} \mathrm{O}_{7}$ (пространственная группа
$F d \overline{3} m)$, представленные в работе Гектора [15]. Допирование моделировалось путем замещения в ячейке $\mathrm{Bi}_{4} \mathrm{Ti}_{4} \mathrm{O}_{14}$ атома висмута (титана) атомом $\mathrm{Sc}$ или In, что соответствует замещению 25 at.\%. В наших расчетах были использованы энергия cut-off в $400 \mathrm{eV}$ и $k$-сетка размером $8 \times 8 \times 8$. Оптимизация параметров решетки и координат ионов проводилась до тех пор, пока остаточные силы на атомах не становились меньше $0.5 \mathrm{meV} / \AA ̊$. После проведения оптимизации геометрии выполнены расчеты полной и парциальных плотностей состояний, зонной структуры и оптических параметров для рассматриваемых моделей пирохлоров.

\section{4. Результаты и обсуждение}

4.1. Синтез и аттестация $\mathrm{Bi}_{1.5} M_{0.5} \mathrm{Ti}_{2} \mathrm{O}_{7}$, $\mathrm{Bi}_{2} \mathrm{Ti}_{1.5} M_{0.5} \mathrm{O}_{7}(M=\mathrm{Sc}, \mathrm{In})$. Синтез $\mathrm{Sc}$-, In-замещенных титанатов висмута проводился твердофазным способом. Согласно результатам рентгенофазового анализа, в ходе термообработки соединений номинального состава $\mathrm{Bi}_{2} \mathrm{Ti}_{1.5} M_{0.5} \mathrm{O}_{7}(M=\mathrm{Sc}, \mathrm{In})$ образуются твердые растворы $\mathrm{Bi}_{4-x} M_{y} \mathrm{Ti}_{3-z} \mathrm{O}_{12-\delta}$ с примесью оксида допируемого элемента $\mathrm{M}_{2} \mathrm{O}_{3}(M=\mathrm{Sc}, \mathrm{In})$. Формирования соединений со структурой типа пирохлора не происходит. В случае $\mathrm{Bi}_{1.5} M_{0.5} \mathrm{Ti}_{2} \mathrm{O}_{7}(M=\mathrm{Sc}, \mathrm{In})$ по данным рентгенофазового анализа (рис. 1) и сканирующей электронной микроскопии (СЭМ) (рис. 2) получены однофазные соединения, со структурой типа пирохлора (ICSD 50983). По данным EDS-анализа (табл. 1) установлено, что после термообработки в ходе твердофазного синтеза исходное соотношение элементов сохраняется.

Методом ДСК выявлено отсутствие структурных фазовых переходов при нагревании на воздухе до точки перитектического плавления $1278^{\circ} \mathrm{C}$ для $\mathrm{Bi}_{1.5} \mathrm{Sc}_{0.5} \mathrm{Ti}_{2} \mathrm{O}_{7}$ и $1287^{\circ} \mathrm{C}$ для $\mathrm{Bi}_{1.5} \mathrm{In}_{0.5} \mathrm{Ti}_{2} \mathrm{O}_{7}$.

Распределение атомов допанта (Sc, In) по кристаллографическим позициям структуры пирохлора определе-

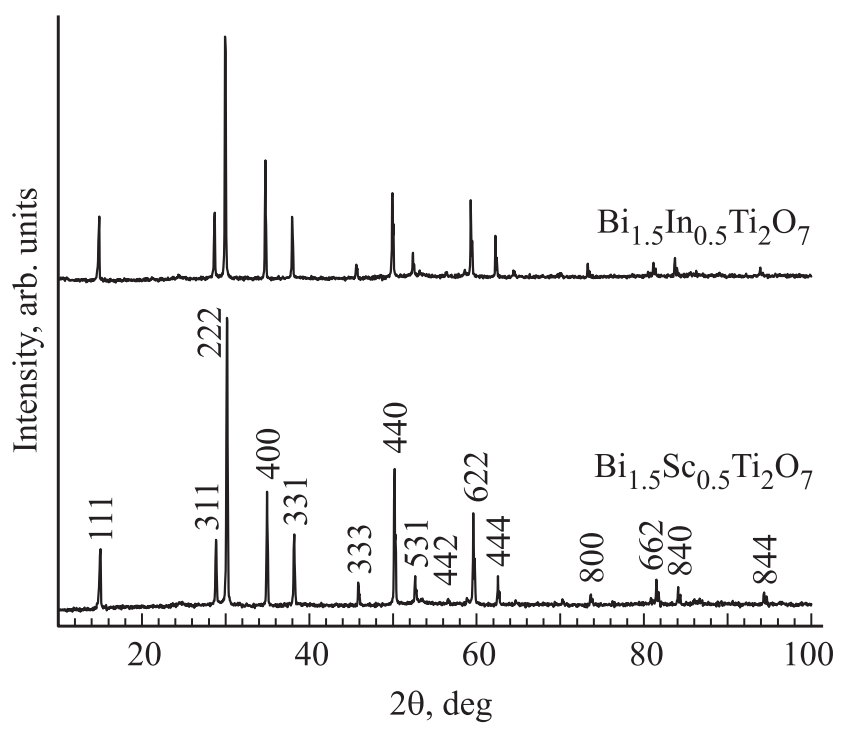

Рис. 1. Рентгенограммы образцов $\mathrm{Bi}_{1.5} M_{0.5} \mathrm{Ti}_{2} \mathrm{O}_{7}(M=\mathrm{Sc}, \mathrm{In})$. 

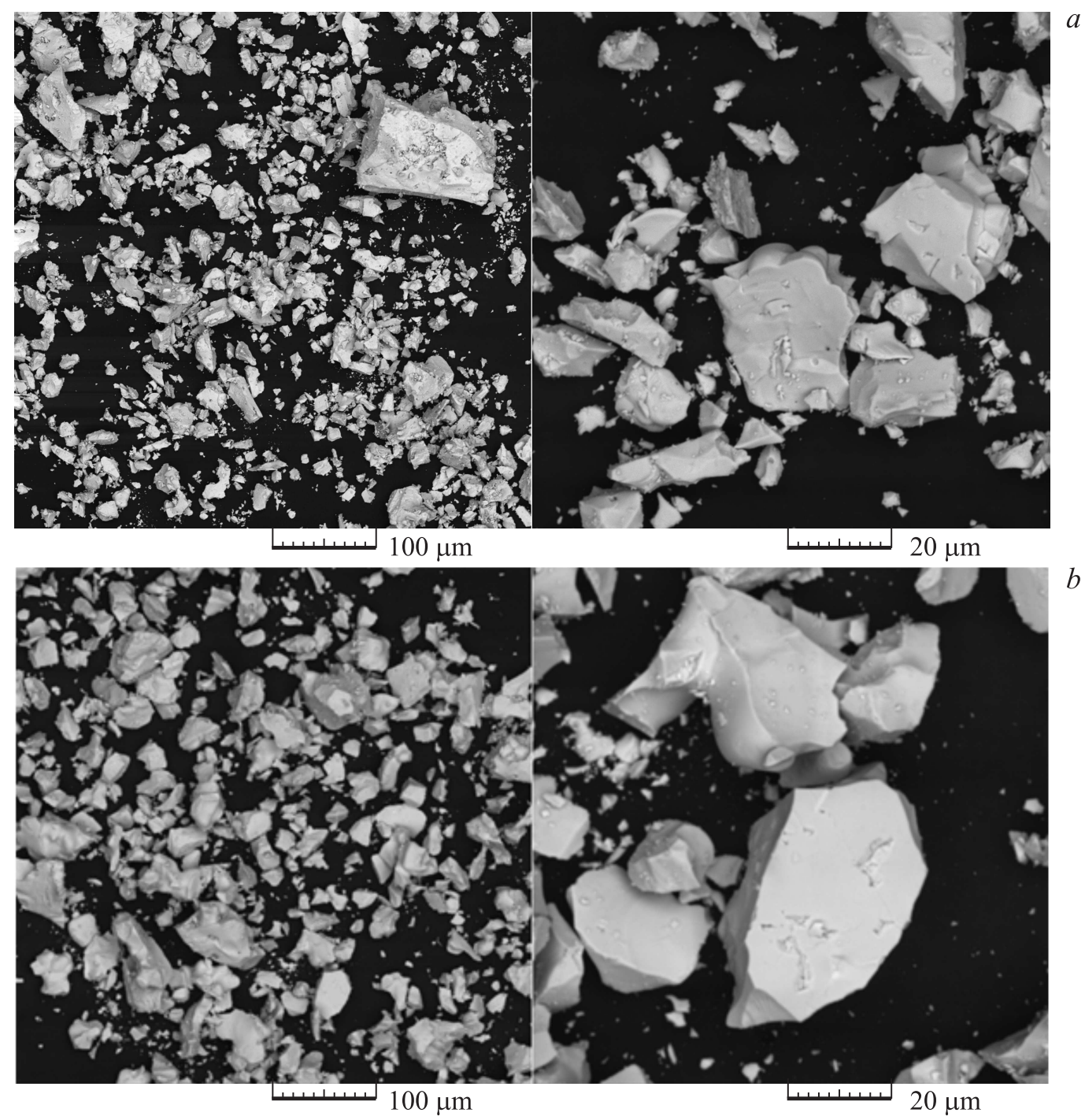

Рис. 2. СЭМ-изображения образцов $\mathrm{Bi}_{1.5} \mathrm{Sc}_{0.5} \mathrm{Ti}_{2} \mathrm{O}_{7}(a)$ и $\mathrm{Bi}_{1.5} \operatorname{In}_{0.5} \mathrm{Ti}_{2} \mathrm{O}_{7}(b)$.

Таблица 1. Исходный и экспериментально определенный составы образцов после синтеза

\begin{tabular}{c|c}
\hline Исходный состав & $\begin{array}{c}\text { Расчетное значение } \\
\text { по данным EDS }\end{array}$ \\
\hline $\mathrm{Bi}_{1.5} \mathrm{Sc}_{0.5} \mathrm{Ti}_{2} \mathrm{O}_{7}$ & $\mathrm{Bi}_{1.65} \mathrm{Sc}_{0.48} \mathrm{Ti}_{2} \mathrm{O}_{7-\delta}$ \\
$\mathrm{Bi}_{1.5} \mathrm{In}_{0.5} \mathrm{Ti}_{2} \mathrm{O}_{7}$ & $\mathrm{Bi}_{1.67} \mathrm{In}_{0.49} \mathrm{Ti}_{2} \mathrm{O}_{7-\delta}$
\end{tabular}

но на основе структурного анализа рентгенограмм и сопоставления пикнометрической и рассчитанной плотностей соединений. Для проведения полнопрофильного анализа использовались данные порошковой дифракции рентгеновских лучей синтезированных образцов. Заселенности атомных позиций фиксировались в соответствии с элементным составом рассматриваемых моделей. Кроме того, изначально фиксировался параметр теплового смещения для атома кислорода $\mathrm{O}^{\prime}$ [15]. Лучшие результаты обработки дифрактограмм замещенных
Таблица 2. Результаты полнопрофильной обработки дифрактограмм

\begin{tabular}{|c|c|c|c|c|c|c|}
\hline Атом & Положение & $x$ & $y$ & $z$ & $B_{\text {iso }}, \AA^{2}$ & Заселенность \\
\hline \multicolumn{7}{|c|}{$\mathrm{Bi}_{1.5} \mathrm{Sc}_{0.5} \mathrm{Ti}_{2} \mathrm{O}_{7}$} \\
\hline $\mathrm{Bi} / \mathrm{Sc}$ & $16 c$ & 0 & 0 & 0 & 1.47 & $0.75 / 0.25$ \\
\hline $\mathrm{Ti} / \mathrm{Sc}$ & $16 d$ & $1 / 2$ & $1 / 2$ & $1 / 2$ & 1.14 & $1 / 0$ \\
\hline $\mathrm{O}$ & $48 f$ & $1 / 8$ & $1 / 8$ & 0.437 & 2.55 & 1 \\
\hline $\mathrm{O}^{\prime}$ & $8 a$ & $1 / 8$ & $1 / 8$ & $1 / 8$ & 0.1 & 1 \\
\hline \multicolumn{7}{|c|}{$a=10.3105 \AA, R_{\mathrm{p}}=5.32 \%, R_{\mathrm{wp}}=6.99 \%, \chi^{2}=2.91$} \\
\hline \multicolumn{7}{|c|}{$\mathrm{Bi}_{1.5} \mathrm{In}_{0.5} \mathrm{Ti}_{2} \mathrm{O}_{7}$} \\
\hline $\mathrm{Bi} / \mathrm{In}$ & $16 c$ & 0 & 0 & 0 & 1.17 & $0.75 / 0.25$ \\
\hline $\mathrm{Ti} / \mathrm{In}$ & $16 d$ & $1 / 2$ & $1 / 2$ & $1 / 2$ & 0.57 & $1 / 0$ \\
\hline $\mathrm{O}$ & $48 f$ & $1 / 8$ & $1 / 8$ & 0.437 & 4.30 & 1 \\
\hline $\mathrm{O}^{\prime}$ & $8 a$ & $1 / 8$ & $1 / 8$ & $1 / 8$ & 0.1 & 1 \\
\hline
\end{tabular}

Примечание. $R_{\mathrm{p}}-$ профильный, $R_{\mathrm{wp}}-$ весовой профильный фактор достоверности, $\chi^{2}-$ индикатор точности. 
Таблица 3. Рассчитанные и пикнометрические плотности

\begin{tabular}{c|c|c|c|c}
\hline $\begin{array}{c}\text { Формулы с учетом } \\
\text { распределения допанта }\end{array}$ & $\begin{array}{c}\text { Доля атомов } \mathrm{Sc}(\mathrm{In}) \\
\text { в } A \text {-позиции, } \%\end{array}$ & $\begin{array}{c}\text { Доля атомов } \mathrm{Sc}(\mathrm{In}) \\
\text { в } B \text {-позиции, } \%\end{array}$ & $\rho_{\text {calc }}, \mathrm{g} / \mathrm{cm}^{3}$ & $\rho_{\text {pycn }}, \mathrm{g} / \mathrm{cm}^{3}$ \\
\hline$\left(\mathrm{Bi}_{1.5} \mathrm{Sc}_{0.5} \square_{0}\right) \mathrm{Ti}_{2} \mathrm{O}_{7}$ & 100 & 0 & 6.588 \\
$\left(\mathrm{Bi}_{1.43} \mathrm{Sc}_{0.38} \square_{0.19}\right)\left(\mathrm{Ti}_{1.90} \mathrm{Sc}_{0.10}\right) \mathrm{O}_{6.67}$ & 80 & 20 & 6.274 \\
$\left(\mathrm{Bi}_{1.33} \mathrm{Sc}_{0.22} \square_{0.44}\right)\left(\mathrm{Ti}_{1.78} \mathrm{Sc}_{0.22}\right) \mathrm{O}_{6.22}$ & 50 & 50 & 5.856 \\
$\left(\mathrm{Bi}_{1.5} \mathrm{In}_{0.5} \square_{0}\right) \mathrm{Ti}_{2} \mathrm{O}_{7}$ & 100 & 0 & 6.977 \\
$\left(\mathrm{Bi}_{1.43} \mathrm{In}_{0.38} \square_{0.19}\right)\left(\mathrm{Ti}_{1.90} \mathrm{In}_{0.10}\right) \mathrm{O}_{6.67}$ & 80 & 20 & 6.645 \\
$\left(\mathrm{Bi}_{1.33} \mathrm{In}_{0.22} \square_{0.44}\right)\left(\mathrm{Ti}_{1.78} \mathrm{In}_{0.22}\right) \mathrm{O}_{6.22}$ & 50 & 50 & 6.202 & 6.924
\end{tabular}

Примечание. $\square-$ катионная вакансия.

соединений, определенные на основании величин факторов достоверности $\left(R_{\mathrm{p}}, R_{\mathrm{wp}}, \chi^{2}\right)$, представлены в табл. 2 .

Значения пикнометрической плотности $\mathrm{Bi}_{1.5} \mathrm{Sc}_{0.5} \mathrm{Ti}_{2} \mathrm{O}_{7}$ и $\mathrm{Bi}_{1.5} \mathrm{In}_{0.5} \mathrm{Ti}_{2} \mathrm{O}_{7}$ совпадают с точностью $2 \%$ со значениями рассчитанных плотностей, соответствующих варианту распределения всех атомов допанта в позиции атомов Ві. Полученные результаты согласуются с данными структурного анализа и подтверждают возможность распределения атомов допанта по подрешетке висмута (табл. 3).

4.2. Квантово-химические расчеты.

4.2.1. Энергетика образования и структурные свойства. Оптимизированный методом DFT параметр элементарной ячейки $\mathrm{Bi}_{2} \mathrm{Ti}_{2} \mathrm{O}_{7} \quad(10.3223 \AA)$ хорошо согласуется с экспериментальным значением $10.3591 \AA$ (расхождение 0.4\%), установленным при исследовании структуры стехиометрического пирохлора титаната висмута с помощью нейтронной дифракции при температуре $2 \mathrm{~K}$ [15] (табл. 4).

Значения оптимизированных и экспериментально установленных в ходе данной работы параметров элементарных ячеек допированных соединений со структурой пирохлора также близки (расхождение 1\%) и коррелируют с радиусами ионов-заместителей: $R_{\text {ion }}(\mathrm{Sc})=0.87 \AA, R_{\text {ion }}(\mathrm{In})=0.92 \AA[13]$. Как видно из табл. 4, замещение позиций висмута элементами с

Таблица 4. Параметр элементарной ячейки

\begin{tabular}{c|c|c}
\hline \multirow{2}{*}{ Соединение } & \multicolumn{2}{|c}{$a, \AA$} \\
\cline { 2 - 3 } & Расчет $(0 \mathrm{~K})$ & Эксперимент \\
\hline $\mathrm{Bi}_{2} \mathrm{Ti}_{2} \mathrm{O}_{7}$ & 10.3223 & $10.3591(2 \mathrm{~K})[15]$ \\
$\mathrm{Bi}_{1.5} \mathrm{Sc}_{0.5} \mathrm{Ti}_{2} \mathrm{O}_{7}$ & 10.2086 & $10.3105(298 \mathrm{~K})$ \\
$\mathrm{Bi}_{1.5} \mathrm{In}_{0.5} \mathrm{Ti}_{2} \mathrm{O}_{7}$ & 10.2136 & $10.3271(298 \mathrm{~K})$
\end{tabular}

Таблица 5. Энергии образования фаз

\begin{tabular}{c|c}
\hline Фаза & $\Delta E$, eV/f.u. \\
\hline $\mathrm{Bi}_{1.5} \mathrm{Sc}_{0.5} \mathrm{Ti}_{2} \mathrm{O}_{7}$ & -2.67 \\
$\mathrm{Bi}_{1.5} \mathrm{In}_{0.5} \mathrm{Ti}_{2} \mathrm{O}_{7}$ & -0.25 \\
$\mathrm{Bi}_{2} \mathrm{Ti}_{1.5} \mathrm{Sc}_{0.5} \mathrm{O}_{7}$ & 0.41 \\
$\mathrm{Bi}_{2} \mathrm{Ti}_{1.5} \mathrm{In}_{0.5} \mathrm{O}_{7}$ & 2.84
\end{tabular}

меньшим ионным радиусом приводит к уменьшению параметров элементарной ячейки, что является одним из факторов стабилизации фазы пирохлора титаната висмута.
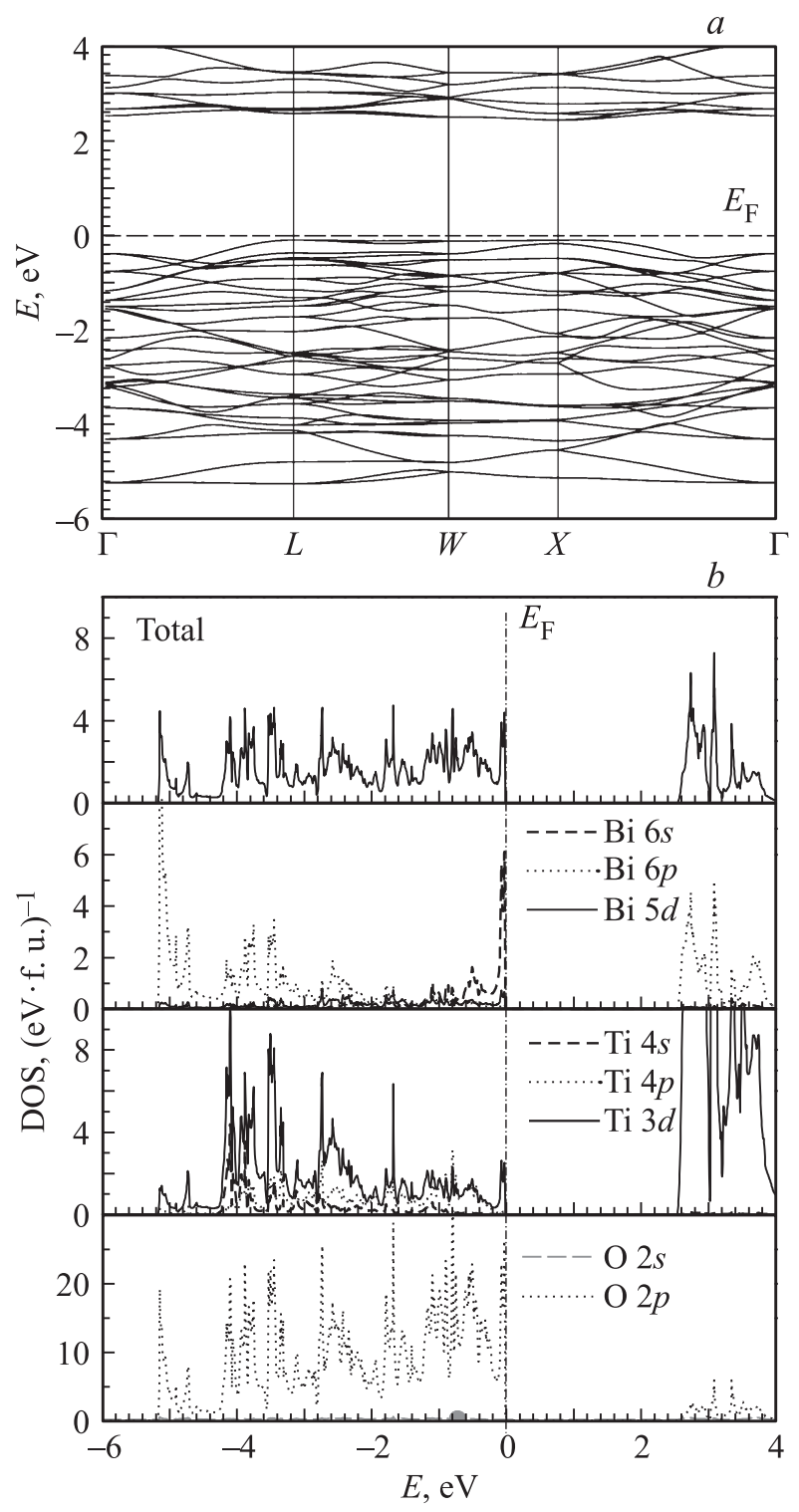

Pис. 3. Энергетические зоны $(a)$ и полная и парциальные плотности электронных состояний $(b)$ для $\mathrm{Bi}_{2} \mathrm{Ti}_{2} \mathrm{O}_{7}$. 
Для оценки энергий образования фаз $\mathrm{Bi}_{2-x} M_{x} \mathrm{Ti}_{2} \mathrm{O}_{7}$ и $\mathrm{Bi}_{2} \mathrm{Ti}_{2-x} M_{x} \mathrm{O}_{7} \quad(M=\mathrm{Sc}, \mathrm{In})$ были определены энергии чистых металлов (кристаллические структуры $\alpha$-Ті и $\alpha$-Sc, пространственная группа $P 63 / m m c$; In, пространственная группа $I 4 / \mathrm{mmm}, \mathrm{Bi}$, пространственная группа $R \overline{3} m H)$. Оценка энергии образования выполнена на основе расчета изменения полной энергии системы в соответствии с уравнениями реакций [41]: $\mathrm{Bi}_{2} \mathrm{Ti}_{2} \mathrm{O}_{7}+0.5 M=\mathrm{Bi}_{1.5} M_{0.5} \mathrm{Ti}_{2} \mathrm{O}_{7}+0.5 \mathrm{Bi}$ и $\mathrm{Bi}_{2} \mathrm{Ti}_{2} \mathrm{O}_{7}+0.5 M=\mathrm{Bi}_{2} \mathrm{Ti}_{1.5} M_{0.5} \mathrm{O}_{7}+0.5 \mathrm{Ti}$, где $M=\mathrm{Sc}, \mathrm{In}$ (табл. 5).
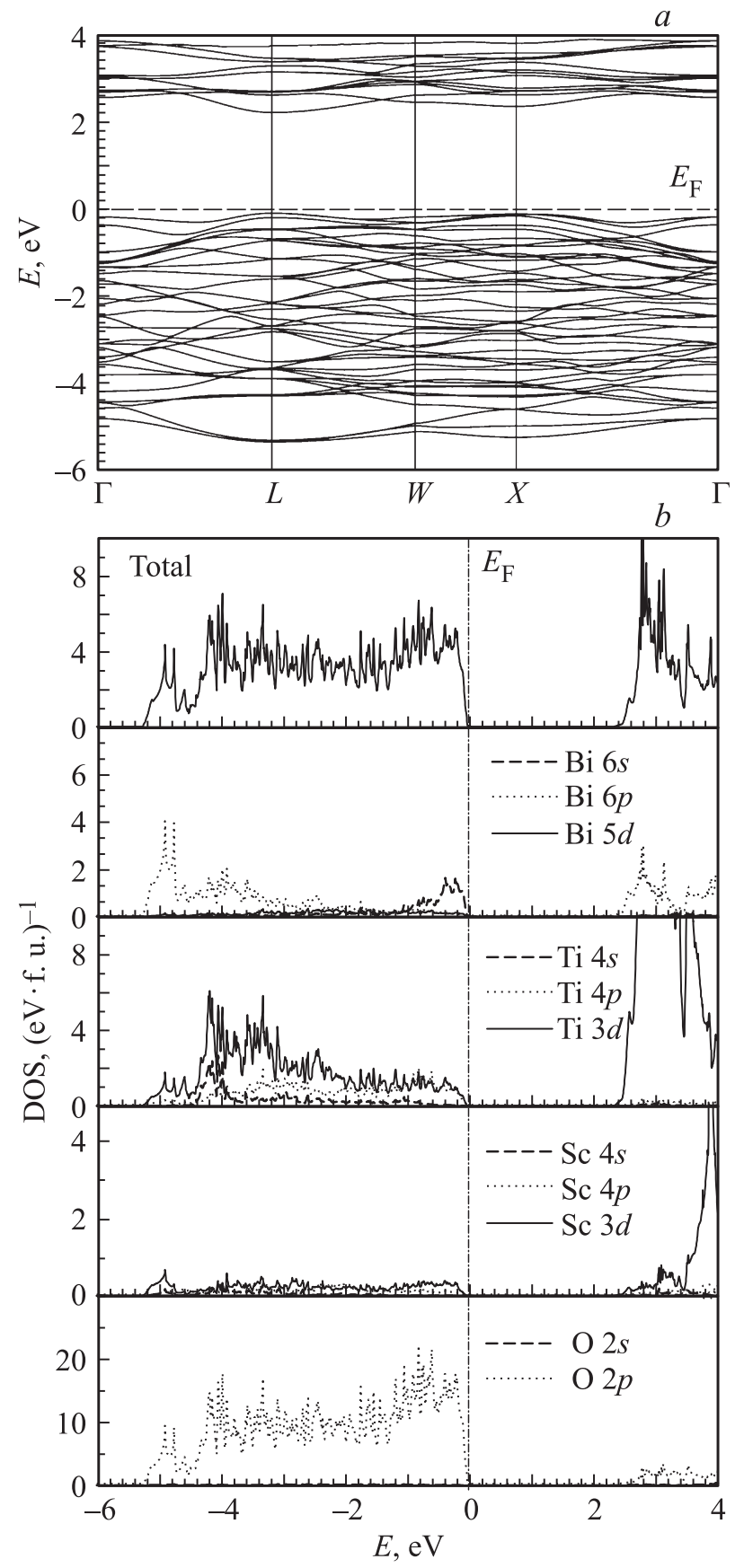

Pис. 4. Энергетические зоны $(a)$ и полная и парциальные плотности электронных состояний $(b)$ для $\mathrm{Bi}_{1.5} \mathrm{Sc}_{0.5} \mathrm{Ti}_{2} \mathrm{O}_{7}$.
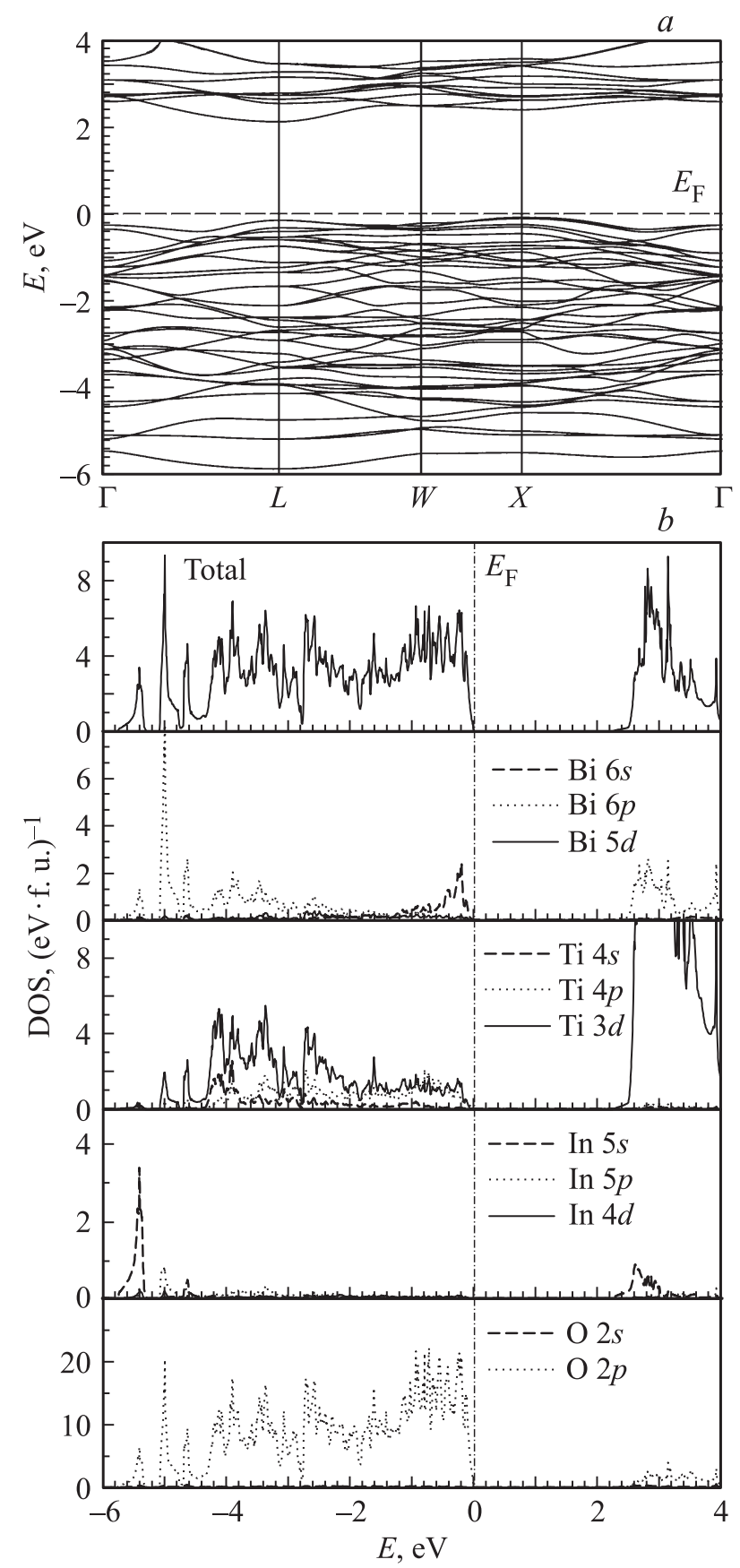

Рис. 5. Энергетические зоны $(a)$ и полная и парциальные плотности электронных состояний $(b)$ для $\mathrm{Bi}_{1.5} \mathrm{In}_{0.5} \mathrm{Ti}_{2} \mathrm{O}_{7}$.

Как видно из этой таблицы, энергии образования фаз для $\mathrm{Bi}_{1.5} \mathrm{Sc}_{0.5} \mathrm{Ti}_{2} \mathrm{O}_{7}$ и $\mathrm{Bi}_{1.5} \mathrm{In}_{0.5} \mathrm{Ti}_{2} \mathrm{O}_{7}$ являются отрицательными, т.е. замещение атомов Вi атомами Sc или In является энергетически предпочтительным процессом. $\mathrm{C}$ другой стороны, для $\mathrm{Bi}_{2} \mathrm{Ti}_{1.5} \mathrm{Sc}_{0.5} \mathrm{O}_{7}$ и $\mathrm{Bi}_{2} \mathrm{Ti}_{1.5} \mathrm{In}_{0.5} \mathrm{O}_{7}$ эти энергии положительны; следовательно, энергетически устойчивых кристаллических структур типа пирохлора данного состава не существует. Этот теоретический расчет полностью совпадает с экспериментальными результатами по синтезу указанных фаз (см. подраздел 4.1). 

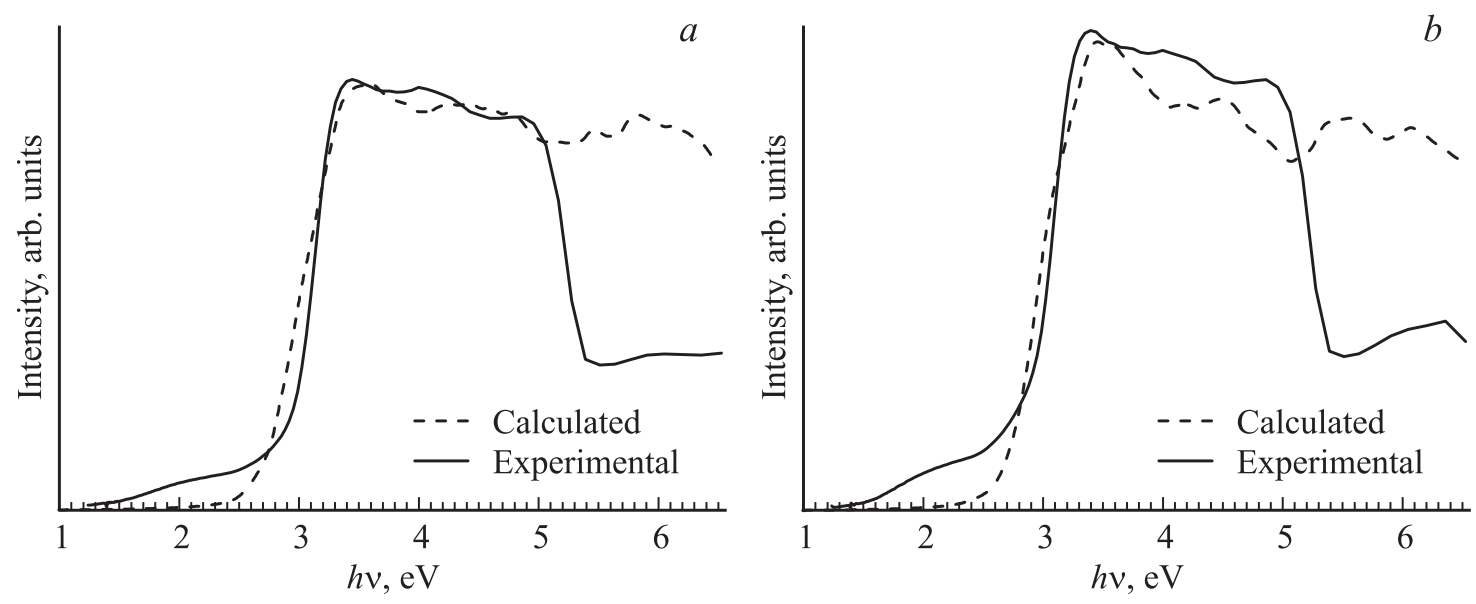

Pис. 6. Спектры оптического поглощения $\mathrm{Bi}_{1.5} \mathrm{Sc}_{0.5} \mathrm{Ti}_{2} \mathrm{O}_{7}(a), \mathrm{Bi}_{1.5} \operatorname{In}_{0.5} \mathrm{Ti}_{2} \mathrm{O}_{7}(b)$.
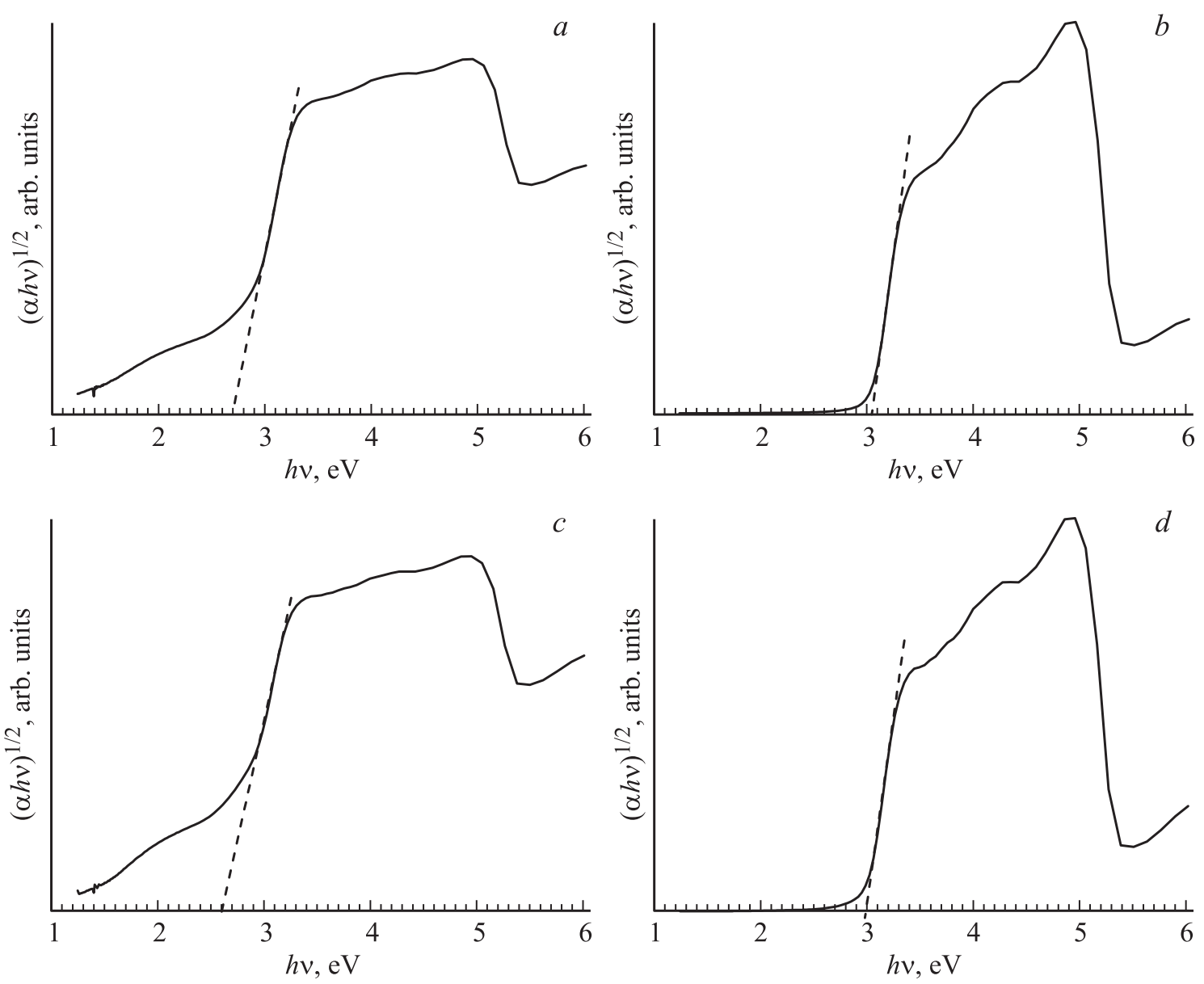

Рис. 7. Построение Тауца по данным оптического поглощения $\mathrm{Bi}_{1.5} \mathrm{Sc}_{0.5} \mathrm{Ti}_{2} \mathrm{O}_{7}(a, b)$ и $\mathrm{Bi}_{1.5} \operatorname{In}_{0.5} \mathrm{Ti}_{2} \mathrm{O}_{7} \quad(c, d)$ для непрямого разрешенного $(a, c)$ и прямого разрешенного $(b, d)$ переходов.

4.2.2. Электронная и зонная структура. На рис. 3-5 представлены энергетические зоны, полные и парциальные плотности электронных состояний. Так, для $\mathrm{Bi}_{2} \mathrm{Ti}_{2} \mathrm{O}_{7}$ наблюдается прямой электронный переход в точке $X$ (рис. $3, a$ ) с величиной запрещенной щели $E_{g}$, равной $2.65 \mathrm{eV}$. Данное значение хорошо согласуется с результатами, полученными ранее в ходе $a b$ initio расчетов электронного строения $\mathrm{Bi}_{2} \mathrm{Ti}_{2} \mathrm{O}_{7}$ (2.6 [29] и $2.85 \mathrm{eV}$ [32]), где был также найден прямой переход для точки $X$. Экспериментальное значение этой величины составляет $2.58-2.83 \mathrm{eV}[7,9,42-45]$.

Основной вклад в формирование валентной зоны $\mathrm{Bi}_{2} \mathrm{Ti}_{2} \mathrm{O}_{7}$ (в пределах $-5.2-0 \mathrm{eV}$ ниже уровня Ферми) вносит перекрывание Ті $3 d$-орбиталей с О $2 p$-орбиталя- 
Таблица 6. Значение величины запрещенной щели по данным оптического поглощения и результатам расчетов DFT

\begin{tabular}{c|c|c|c}
\hline \multirow{2}{*}{ Соединение } & \multicolumn{3}{|c}{$E_{g}, \mathrm{eV}$} \\
\cline { 2 - 4 } & Прямой переход & Непрямой переход & Теория \\
\hline $\mathrm{Bi}_{2} \mathrm{Ti}_{2} \mathrm{O}_{7}$ & \multicolumn{2}{|c|}{$2.58-2.83[7,9,42-47]$} & 2.65 \\
$\mathrm{Bi}_{1.5} \mathrm{Sc}_{0.5} \mathrm{Ti}_{2} \mathrm{O}_{7}$ & 3.05 & 2.70 & 2.42 (прямой переход) \\
$\mathrm{Bi}_{1.5} \mathrm{In}_{0.5} \mathrm{Ti}_{2} \mathrm{O}_{7}$ & 2.97 & 2.60 & 2.31 (непрямой переход)
\end{tabular}

ми с примесью Вi $6 s-$, Вi $6 p$-орбиталей (рис. $3, b$ ). Образование зоны проводимости в диапазоне $2.55-4.2 \mathrm{eV}$ выше уровня Ферми происходит за счет перекрывания О2p-орбиталей с незаполненными Ві $6 p$ - и Ті $3 d$-орбиталями. Установленная в ходе наших расчетов картина электронного строения $\mathrm{Bi}_{2} \mathrm{Ti}_{2} \mathrm{O}_{7}$ близка к полученной в работах других авторов [16,28-30,32].

Как видно из рис. 4, $a$, для $\mathrm{Bi}_{1.5} \mathrm{Sc}_{0.5} \mathrm{Ti}_{2} \mathrm{O}_{7}$ величина запрещенной щели составляет $2.42 \mathrm{eV}$ вдоль направления $L$ (прямой электронный переход). При замещении атомов висмута скандием его $3 d$-состояния участвуют в формировании валентной зоны и зоны проводимости (рис. $4, b$ ) при этом вклад Sc $4 s-$-, Sc $4 p$-состояний в этих зонах несуществен.

Для случая $\mathrm{Bi}_{1.5} \mathrm{In}_{0.5} \mathrm{Ti}_{2} \mathrm{O}_{7}$ в отличие от систем, рассмотренных выше, электронный переход будет непрямым $(X-L)$ со значением энергии $2.31 \mathrm{eV}$ (рис. 5, $a$ ).

Анализ плотностей состояний показал, что In $5 s-$ и In $5 p$-состояния участвуют в формировании валентной зоны и частично зоны проводимости (рис. $5, b$ ), при этом вклад $4 d$-орбиталей незначителен.

Представленные выше результаты по электронной структуре стехиометрического $\mathrm{Bi}_{2} \mathrm{Ti}_{2} \mathrm{O}_{7}$ и замещенных $\mathrm{Bi}_{1.5} \mathrm{Sc}_{0.5} \mathrm{Ti}_{2} \mathrm{O}_{7}$ и $\mathrm{Bi}_{1.5} \mathrm{In}_{0.5} \mathrm{Ti}_{2} \mathrm{O}_{7}$ получены для спинограниченного базиса. Расчеты, выполненные в спинполяризованном приближении, показывают, что в данных системах отсутствует образование локальных магнитных моментов на атомах.

4.3. Оптические свойства. Расчет спектров поглощения в рамках пакета VASP мы провели методом DFPT (density functional perturbation theory) при помощи преобразования Крамерса-Кронинга [46], интегрируя мнимую часть диэлектрической функции. Представленные на рис. 6 спектры показывают хорошее совпадение экспериментальные данных и результатов теоретических расчетов в области энергий $1-5 \mathrm{eV}$.

Потолок валентной зоны и дно зоны проводимости в допированных скандием (рис. 4, $a$ ) и индием (рис. 5, $a$ ) пирохлорах имеют более одного экстремального значения, поэтому оптические межзонные переходы могут быть как прямыми, так и непрямыми.

По экспериментальным данным для поглощения выполнено построение Тауца (рис. 7) в координатах $(\alpha h \omega)^{n}-h v$, где $n=1 / 2$ и 2 относятся к непрямому разрешенному и прямому разрешенному переходам соответственно $[47,48]$.

Полученные значения оптической ширины запрещенной зоны представлены в табл. 6 в сравнении со значениями ширины запрешенной зоны, рассчитанными методом DFT.

По величине энергии запрещенной щели данные соединения можно отнести к классу диэлектриков. Энергии квантов видимого света $(\lambda \geq 380 \mathrm{~nm})$ окажется достаточно для фотовозбуждения электронов из валентной зоны в зону проводимости. Следовательно, рассматриваемые соединения могут быть перспективны в качестве фотокатализаторов в видимой области спектра при их получении в наноразмерном состоянии.

\section{5. Заключение}

Проведено сопоставление результатов исследования структурных, оптических параметров допированных скандием, индием (25 at.\%) титанатов висмута со структурой типа пирохлора с данными $a b$ initio квантовохимических расчетов структурных, электронных и оптических свойств. Показано, что замещение допантом позиций висмута энергетически выгодно и приводит к образованию устойчивой кристаллической структуры типа пирохлора. По результатам расчета электронной и зонной структуры определены величины запрещенной щели для прямого $\left(\mathrm{Bi}_{2} \mathrm{Ti}_{2} \mathrm{O}_{7}-2.65 \mathrm{eV}\right.$, $\left.\mathrm{Bi}_{1.5} \mathrm{Sc}_{0.5} \mathrm{Ti}_{2} \mathrm{O}_{7}-2.42 \mathrm{eV}\right)$ и непрямого электронного перехода $\left(\mathrm{Bi}_{1.5} \mathrm{In}_{0.5} \mathrm{Ti}_{2} \mathrm{O}_{7}-2.31 \mathrm{eV}\right)$, которые коррелируют с экспериментальными результатами изучения оптического поглощения.

\section{Список литературы}

[1] J.R. Esquivel-Elizondo, B.B. Hinojosa, J.C. Nino. Chem. Mater. 23, 4965 (2011).

[2] Ch.H. Yang, H.T. Wu, D.M. Yang. Mater. Lett. 61, 4166 (2007).

[3] G.W. Hwang, W.D. Kim, Y.S. Min, Y.J. Cho, C.S. Hwang. J. Electrochem. Soc. 153, 20 (2006).

[4] A. Hardy, S.V. Elshocht, C.D. Dobbelaere, J. Hadermann, G. Pourtois, S.D. Gendt, V.V. Afanas'ev, M.K. Van Bael. Mater. Res. Bull. 47, 511 (2012).

[5] I.V. Piir, M.S. Koroleva, Y.I. Ryabkov, D.A. Korolev, N.V. Chezhina, V.G. Semenov, V.V. Panchuk. J. Solid State Chem. 204, 245 (2013).

[6] I.V. Piir, M.S. Koroleva, Y.I. Ryabkov, E.Yu. Pikalova, S.V. Nekipelov, V.N. Sivkov, D.V. Vyalikh. Solid State Ion. 262, 630 (2014).

[7] J. Hou, Sh. Jiao, H. Zhu, R.V. Kumar. J. Solid State Chem. 184, 154 (2011). 
[8] W.F. Yao, H. Wang, X.H. Xu, J.T. Zhou, X.N. Wang, Y. Zhang, S.X. Shang. Appl. Catal. A 259, 29 (2004).

[9] L.Z. Pei, H.D. Liu, N. Lin, H.Y. Yu. J. Alloys Compd. 622 , 254 (2015).

[10] S. Murugesan, V.R. Subramanian. Chem. Commun. 34, 5109 (2009).

[11] M.A. Subramanian, G. Aravamudan, G.V.S. Rao. Prog. Solid State Chem. 15, 55 (1983).

[12] R.A. McCauley. J. Appl. Phys. 51, 290 (1980).

[13] R.D. Shannon. Acta Cryst. A 32, 751 (1976).

[14] I. Radosavljevic, J.S.O. Evans, A.W. Sleight. J. Solid State Chem. 136, 63 (1998)

[15] A.L. Hector, S.B. Wiggin. J. Solid State Chem. 177, 139 (2004).

[16] B.B. Hinojosa, J.C. Nino, A. Asthagiri. Phys. Rev. B 77, 104123 (2008).

[17] O. Merka, D.W. Bahnemann, M. Wark. Catal. Today 225, 102 (2014).

[18] I.V. Piir, N.A. Sekushin, V.E. Grass, Y.I. Ryabkov, N.V. Chezhina, S.V. Nekipelov, V.N. Sivkov, D.V. Vyalikh. Solid State Ion. 225, 464 (2012).

[19] М.С. Королева, И.В. Пийр, Ю.И. Рябков, Д.А. Королев, Н.В. Чежина. Изв. АН. Сер. хим. 2, 410 (2013).

[20] И.В. Пийр, М.С. Королева, Н.А. Секушин, В.Э. Грасс, Ю.И. Рябков. Электрохимия 49, 909 (2013).

[21] М.М. Пискайкина, И.В. Пийр, М.С. Королева, В.А. Белый. Изв. Коми НЦ УрО РАН 21, 22 (2015).

[22] S. Kunej, D. Suvorov. J. Am. Ceram. Soc. 91, 3472 (2008).

[23] S. Kunej, D. Suvorov. J. Am. Ceram. Soc. 92, 959 (2009).

[24] S. Kunej, S.D. Scapin, D. Suvorov. J. Am. Ceram. Soc. 92, 2373 (2009).

[25] S. Kunej, S.D. Scapin, D. Suvorov. J. Am. Ceram. Soc. 95, 1201 (2012).

[26] R. Seshadri. Solid State Sci. 8, 259 (2006).

[27] C.J. Fennie, R. Seshadri, K.M. Rabe. arXiv:0712. 1846 (2007).

[28] W. Wei, Y. Dai, B. Huang. J. Phys. Chem. C 113, 5658 (2009).

[29] S. Murugesan, M.N. Huda, Y. Yan, M.M.A. Jassim, V. Subramanian. J. Phys. Chem. C 114, 10598 (2010).

[30] C.H. Patterson. Phys. Rev. B. 82, 155103 (2010).

[31] D.P. Shoemaker, R. Seshadri, M. Tachibana, A.L. Hector. Phys. Rev. B 84, 064117 (2011).

[32] Q. Fu, T. He, J.L. Li, G.W. Yang. J. Appl. Phys. 111, 124306 (2012).

[33] C.L. Mayfield, M.N. Huda. J. Cryst. Growth 444, 46 (2016).

[34] А.Г. Краснов, М.М. Пискайкина, И.В. Пийр. ЖОХ 86, 177 (2016).

[35] А.Г. Краснов, М.М. Пискайкина, И.В. Пийр. Химия в интересах устойчивого развития 24, 687 (2016).

[36] J. Rodriguez-Carvajal. Physica B 192, 55 (1993).

[37] ГОСТ 2211-65 Огнеупоры и огнеупорное сырье. Методы определения плотности. Введ. 30.06.1966. Изд-во стандартов, М. (1994). 12 с.

[38] G. Kresse, J. Furthmuller. Phys. Rev. B 54, 11169 (1996).

[39] G. Kresse, D. Joubert. Phys. Rev. B 59, 1758 (1999).

[40] J.P. Perdew, S. Burke, M. Ernzerhof. Phys. Rev. Lett. 77, 3865 (1996).

[41] A. Slassi. Mater. Sci. Semicond. Proc. 32, 100 (2015).

[42] Z. Bian, Y. Huo, Y. Zhang, J. Zhu, Y. Lu, H. Li. Appl. Catal. B 91, 247 (2009).
[43] B. Allureda, S. Dela Cruz, T. Darlinga, M.N. Hudac, V. Subramanian. Appl. Catal. B 144, 261 (2014).

[44] S. Gupta, L. De Leon, V.R. Subramanian. Phys. Chem. Chem. Phys. 16, 12719 (2014).

[45] A. McInnes, J.S. Sagu, K.G.U. Wijayantha. Mater. Lett. 137, 214 (2014).

[46] M. Gajdoš, K. Hummer, G. Kresse, J. Furthmüller, F. Bechstedt. Phys. Rev. B 73, 045112 (2006).

[47] M.D. Scafetta, A.M. Cordi, J.M. Rondinelli, S.J. May. J. Phys.: Cond. Matter 26, 505502 (2014).

[48] M.J. Pinzon, J.E. Alfonso, J.J. Olaya. Thin Solid Films 549, 8 (2013). 